\title{
Evaluación in vitro de extractos de hojas y flores de llama del bosque (Spathodea campanulata B.) sobre la broca del café (Hypothenemus hampei F.)
}

In vitro evaluation of African tulip (Spathodea campanulata B.) flowers and leaves extracts on berry borer of coffee (Hypothenemus hampei F.)

Reynaldo Alarcón-Noguera'

Guillermo Penieres-Carrillo²

Fecha de recepción: 27 de noviembre del 2012

Fecha de aprobación: 20 de marzo del 2013

Alarcón-Noguera, R; Penieres-Carrillo, G. Evaluación in vitro de extractos de hojas y flores de llama del bosque (Spathodea campanulata B.) sobre la broca del café (Hypothenemus hampei F.). Tecnología en Marcha. Vol. 26, № 3. Pág 39-49

I Centro Universitario de Suroccidente, USAC. Carrera Agronomía Tropical. Mazatenango, Suchitepéquez, Guatemala. Ing. Agr. M.Sc. reymazate@gmail.com

2 Facultad de Estudios Superiores, Cuautitlán, Campo I. Cuatitlanlxcally, UNAM. México. Ph.D. penieres@unam.mx. 


\section{Palabras clave}

Llama del bosque; Spathodea campanulata; extracto de hojas y flores; broca del café; efecto insecticida; porcentaje de mortalidad

\section{Resumen}

La broca del café (Hypothenemus hampei F.) es la plaga de mayor importancia económica en la caficultura de Guatemala, ya que afecta el rendimiento y la calidad del grano. Entre los métodos de manejo de este insecto está el uso de extractos de hojas y flores de llama del bosque (Spathodea campanulata B.), un árbol con propiedades insecticidas y repelente de insectos utilizado en la medicina popular. El néctar y los extractos de hojas, tallos y flores contienen sustancias tóxicas para los insectos, tales como ácidos orgánicos, esteroles, carbohidratos y triterpenos. En los ensayos in vitro, los extractos de hojas a 0,5 (TI), I,0 (T2) y I,5 (T3) mg/ml de solución tuvieron actividad insecticida, presentando porcentajes de mortalidad de las brocas de 60, 70 y $80 \%$ respectivamente a las 72 horas de aplicación, aunque estadísticamente fueron iguales al insecticida Endosulfán (T4), que provocó el 100\% de mortalidad a las 24 horas de la aplicación, pero diferentes al control negativo (T5), que alcanzó el 20\% de mortalidad a las 72 horas. Los extractos de flores a I y $1.5 \mathrm{mg} / \mathrm{ml}$ de solución provocaron una mortalidad del 100\% de las brocas, lo cual corresponde al mismo efecto del Endosulfán 24 horas después de las aplicaciones, mientras que el extracto a $0.5 \mathrm{mg} /$ $\mathrm{ml}$ presentó el $70 \%$ de mortalidad a las 72 horas. Estadísticamente, el Endosulfán es igual al TI, T2 y T3 pero diferente al control negativo de agua-etanol al 25\% (T5), con un 40\% de mortalidad de brocas. Con base en los resultados anteriores, los extractos de hojas y flores de llama del bosque ofrecen una alternativa para el manejo integrado de la broca del café. Se recomiendan investigaciones in vitro, en invernaderos y en el campo.

\section{Key words}

Llama del bosque; Spathodea campanulata; flowers and leaves extract; berry borer of coffee; insecticide effect; mortality percent

\section{Abstract}

The berry borer of coffee (Hypothenemus hampei F.) is the most important Guatemalan coffee pest. It affects the yield and coffee quality. Among the methods to control pest, we have the use of flowers and leaves extracts from African Tulip (Spathodea campanulata B.). This is a tree with insect repellent and insecticide properties and is used in popular medicine. The nectar and flowers or leaves extracts contain insecticide substances such as organic acids, sterols, carbohydrates and triterpenos. In the in vitro essay, the leaves extracts to $0.5,1.0$ and $1.5 \mathrm{mg} / \mathrm{ml}$ of solution showed insecticide activity, obtained percent of mortality the berry borer of coffee of 60,70 and $80 \%$ respectively after 72 hours of application. Moreover, statistically were the same as Endosulfán that gave 100\% of mortality since 24 hours to application, but different to negative control, that reached $20 \%$ of mortality after 24 hours of the application. The flowers extract to 1.0 and $1.5 \mathrm{mg} / \mathrm{ml}$ of solution obtained $100 \%$ of the berry borer of coffee mortality; the same effect of Endosulfán after 24 hours of application, while the extract to $0.5 \mathrm{mg} / \mathrm{ml}$ showed $70 \%$ of mortality after 72 hours. Statistically Endosulfán was the same to TI,T2 and T3 but different to negative control of ethanol-water to $25 \%$ (T5), which showed $40 \%$ of berry borer of coffee mortality.

On the base of these results, flowers and leaves extracts of African tulip offer an alternative for the integrated management of the berry borer of coffee. In vitro research, in greenhouses or outdoors, is recommended. 


\section{Introducción}

Las plagas de los cultivos agrícolas de Centroamérica constituyen una limitante severa para la producción de granos, frutas y vegetales. La región es una de las que mayor uso de plaguicidas aplica por área agrícola para reducir los daños de los insectos, patógenos y otros organismos bióticos, que pueden disminuir hasta en un 40\% la cosecha (Andrews \& Quezada, 1989). Centroamérica cuenta con la estadística de mayor uso de plaguicidas por año, sobrepasando los dos kilos por persona. Como resultado, la cantidad de envenenamientos agudos por plaguicidas también es alta y excede los 6000 casos anuales. Se estima que más de cuatro millones de personas están expuestas a pesticidas peligrosos (OEA, 2002).

Los insectos en gran cantidad afectan los cultivos en diferentes estados de desarrollo. La forma tradicional de controlar los insectos es mediante el uso de insecticidas químicos, los cuales se han aplicado unilateralmente en forma excesiva, repetitiva y sin un manejo adecuado, llegando a generar problemas de resistencia de las plagas de insectos a los insecticidas, lo que ha ocasionado el surgimiento de nuevas plagas, problemas de contaminación ambiental e intoxicaciones agudas, crónicas y detección de residuos en alimentos (García, 1997), así como un considerable aumento en el costo de producción del cultivo, especialmente en granos básicos y hortalizas.

En el contexto del manejo integrado de plagas, los plaguicidas deben ser el último recurso debido a sus repercusiones en otros organismos y en la salud humana. Por lo tanto, es necesario investigar y desarrollar sistemas alternativos de control de plagas, como es el uso de insecticidas botánicos, los cuales son más amigables con el medio ambiente y pueden llegar a ser elaborados por los mismos agricultores (Hellpap, 2000).

Entre las plagas de cultivos más importantes en Guatemala está la broca del café, que es un insecto del orden Coleoptera, familia Scolytidae, especie Hypothenemus hampei Ferr (ANACAFE, 1998). La broca se encuentra en todas las zonas cafetaleras del país, lo que afecta el rendimiento (conversión) y la calidad del café. La hembra inicia su perforación en la corona del grano, abre una galería en la semilla y deposita sus huevecillos. El mayor daño es causado cuando el grano está en estado de semiconsistencia, ofreciendo un sustrato adecuado para la oviposición, alimentación de adultos y el desarrollo de los estados inmaduros (huevo, larva y pupas) del insecto. Este daño da como resultado la pérdida de peso del grano (Hill, 2008). El insecto adulto se presenta en la Figura I.

En Guatemala, el principal método de control de la broca lo constituye el uso de Endosulfán, un insecticida organoclorado muy efectivo pero residual y persistente, que se encuentra en proceso de prohibición en el mercado regional por sus efectos adversos para el medio ambiente. Entre las alternativas de control de la broca del café están los extractos naturales de plantas con actividad insecticida. En algunos estudios realizados en Brasil se determinó que el néctar del mucílago de las flores del árbol conocido como llama del bosque o tulipán africano (Spathodea campanulata Beauv), causa la muerte de insectos, especialmente abejas, avispas, moscas y hormigas (Trigo \& Santos, 2000).

En Guatemala no se dispone de estudios sobre el efecto insecticida de los extractos de hojas y flores de llama del bosque sobre plagas de insectos. Con base en los antecedentes de uso de esta planta en otros países, los extractos y el néctar pueden constituir una alternativa a los insecticidas químicos en el combate de las plagas de insectos. Es por ello que en el presente trabajo se evaluó el efecto insecticida de los extractos de hojas y flores de llama del bosque para el control de la broca del café.

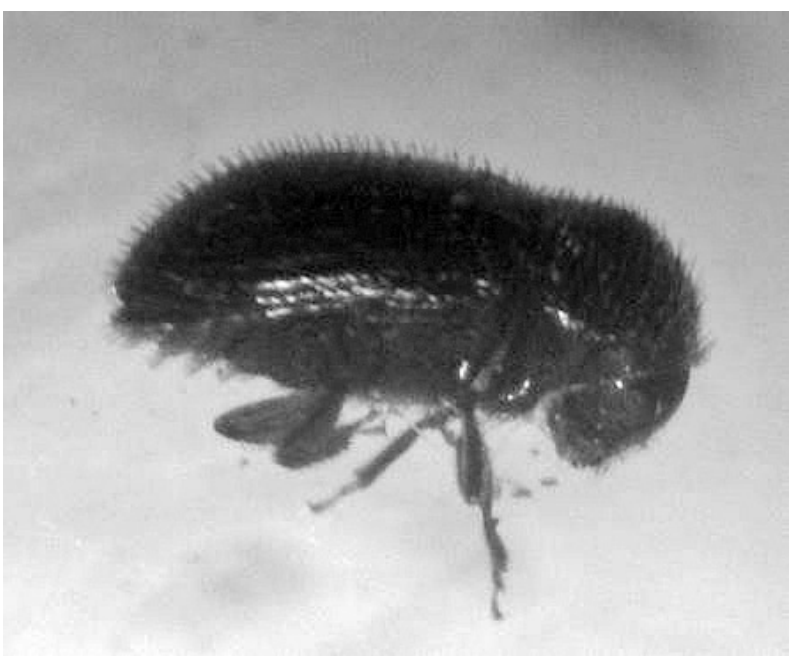

Figura I. Adulto de broca del café (H. hampei F.). Fotografía de Alarcón, 2012. 


\section{Distribución, composición y situación de $S$. campanulata y su uso para el control de plagas de insectos}

\section{Distribución de S. campanulata}

La especie S. campanulata pertenece a la familia Bignoniaceae y se conoce popularmente como llama del bosque o tulipán africano. Es nativa de África Occidental e introducida en América y Asia. El tulipán africano se ha plantado exitosamente como una especie ornamental a través de la zona tropical húmeda (Francis, 1990). En Colombia se ha usado como sombra de cafetales pero es inferior a otros árboles usados con este propósito. Es frecuente su uso como poste en cercas vivas, también para ayudar a la rehabilitación de tierras perturbadas debido a su rápido crecimiento (Semicol, 2010).

En la región de origen de este árbol, sus flores son polinizadas por pájaros, como colibríes o chupamiel de la especie Eupetomena macroura (Trochilidae), que frecuentemente visitan las flores y recolectan el néctar directamente de la flor o perforan los botones florales en la base de la corola. En Panamá se ha determinado que las flores son polinizadas por murciélagos (Flach, 2005).

\section{Composición del néctar de las flores}

El néctar de las flores (ver figura 2) es utilizado para atraer insectos, hormigas y larvas. El néctar es secretado por las glándulas llamadas nectarios y se puede encontrar en los tejidos florales, especialmente en

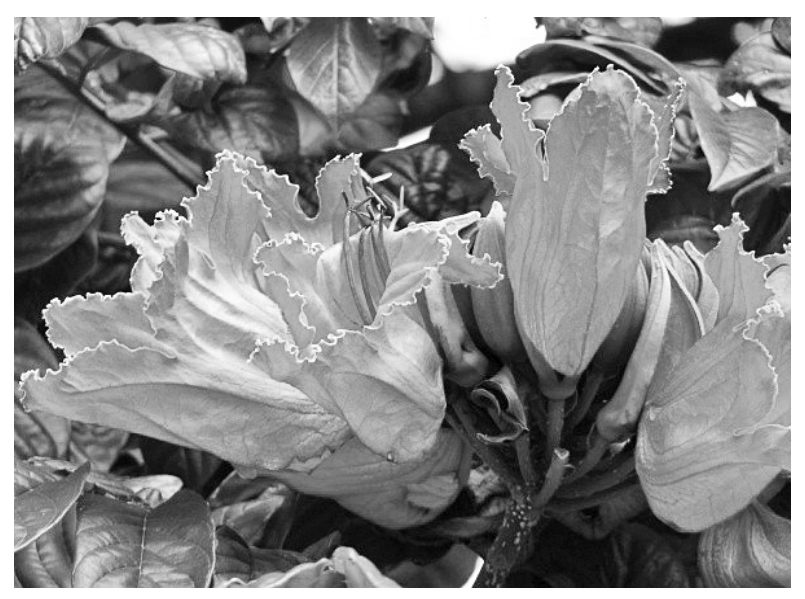

Figura 2. Hojas y flores de llama del bosque. Fotografía de Alarcón, 2012. el cáliz, corola, estambres y carpelo (Flach, 2005). La toxicidad del néctar de las flores está asociada a la defensa de las plantas contra el robo de polen o néctar y está controlada para no afectar a sus polinizadores, como los pájaros chupamiel.

El néctar está constituido por carbohidratos, proteínas, aminoácidos e iones inorgánicos que producen la toxicidad; por otro lado, es aromático y atrayente para algunos organismos (Davis et al., 1998).

Noguela et al. (1990) son los investigadores que más han estudiado los metabolitos de esta planta. Estos autores indican que a partir del extracto clorofórmico del tallo se lograron aislar triterpenos: ácido 3- $\beta$-acetoxioleanólico (SCI), ácido siaresinólico (SC2), 3- $\beta$-acetoxi- I 2-hidroxiolean-28, I 3-olidio (SC3) y ácido oleanólico (SC4). Los investigadores también aislaron del tallo el triterpeno ácido espatódico (SC5) y el 3-O- $\beta$-D-glucopiranosideo sitosterol (SC6) De los extractos en acetato de etilo de las hojas se aisló el hidroxiesterol espatodol (SC7) y el $\beta$-sitosterol (SC8).

Makinde et al. (1987) aislaron algunos compuestos activos, siendo ellos el acido 3- $\beta$-hidroxi urs-12en-28-oico (ácido ursólico) ( $\mathrm{SClO}$ ) y dos de sus derivados: el ácido 3- $\beta$-hidroxiurs-12,19-dien28-oico (ácido tomentosólico (SCII) y el ácido 3- $\beta$-dihidroxiurs I2-en-28-óico (SCI2). Flach (2005) reporta que el néctar de las flores tiene constituyentes volátiles, carbohidratos y compuestos secundarios. En la fracción acuosa del néctar de las flores de $S$. campanulata, encontró evidencia de carbohidratos como sorbona, fructosa, glicosa, manosa y sacarosa. También identificó en el néctar a la alfa-amirina y la beta-amirina, cicloeucalenol, colesterol, estigmasterol y otros triterpenos presentes en las muestras.

\section{Mortalidad de insectos y efectos medicinales de los extractos}

En estudios realizados en Brasil se encontraron más de 200 insectos muertos en flores de S. campanulata recolectadas en los árboles, la mayoría eran abejas meliponas, moscas y hormigas (Portugal-Araujo, 1963). En observaciones de campo, también en Brasil, se determinó que durante el día, cuando las flores se abren (antesis), muere el 96,8\% de insectos; en el segundo día la mortalidad disminuye mucho $(2,3 \%)$ y del tercero al quinto día la mortalidad de 
insectos cae al 0,3\%. Entre los insectos muertos están las abejas Scaptotrigona postica, que fueron encontradas con polen de la planta en sus patas. Al examinar 445 flores de llama del bosque, se encontraron 345 insectos muertos (97\% meliponinae, en su mayoría S. postica; otros fueron: I,7\% díptera y vespidae, $1 \%$ formicidae y $0,3 \%$ orthoptera). Los himenópteros y dípteros eran muy pequeños, con dimensiones de 0,5 a 1,0cm, y los ortópteros de aproximadamente $2 \mathrm{~cm}$ de largo. En cuanto a estudios del néctar de la planta, se determinó que en el mucílago contenido en los botones florales y en las flores jóvenes puede estar disuelto el néctar y ser el responsable de la muerte de los insectos (Trigo y Santos, 2000).

Otros investigadores, como Makinde et al. (1998), reportan que los extractos hexánicos, clorofórmicos y acuosos de la cáscara del tallo fueron investigados en su actividad contra Plasmodium berghei berghei en ratas. Encontraron que los extractos hexánicos y clorofórmicos suprimieron la actividad entre 22 a $80 \%$ y 52 a $74 \%$ respectivamente, quedando sometidos a dosis de 50 a $400 \mathrm{mg} / \mathrm{kg}$.

Los extractos acuosos y butanólicos de la cáscara del tallo también presentaron actividad hipoglicémica en ratas, pero no tuvieron influencia en el nivel de insulina en ratas diabéticas. Los extractos de la corteza también han presentado efectos anti $\mathrm{VIH}$ (Das et al., 2009).

\section{Características del insecticida Endosulfán}

El Endosulfán es un insecticida organoclorado no sistémico de amplio campo de acción, de gran persistencia y con amplia actividad incluso sobre

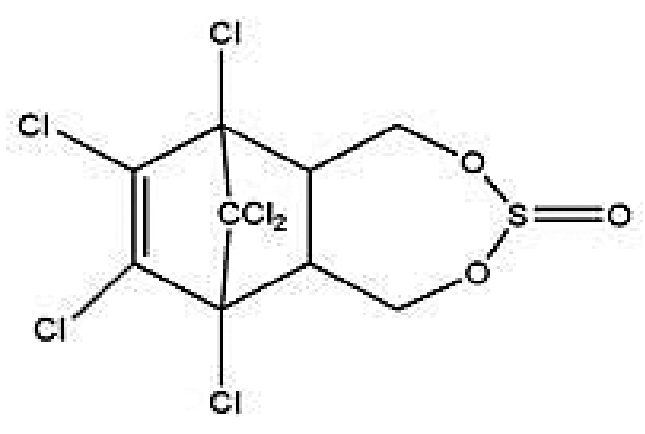

Figura 3. Estructura química del Endosulfán. algunos ácaros. Actúa por ingestión, contacto e inhalación, es residual y no penetrante. Afecta el sistema nervioso por inhibición antagónica del mesoinositol. Es el insecticida más utilizado para el control de la broca del café. Desde el punto de vista químico, está formado por una mezcla de dos estereoisomeros: alfa endosulfán, con 64-67\% de la composición técnica y $\beta$ endosulfán con $29-32 \%$ de la composición técnica. En la Figura 3 se presenta la estructura química del $\mathrm{C} 9 \mathrm{H} 6 \mathrm{Cl} 6 \mathrm{O} 3 \mathrm{~S}$ (sulfito de I ,2.3.4,7,7-hexacloro-9, I 0-trinorborn-2-en-5,6-ilendimetilo), ambos componentes se degradan por oxidación formando ensodulfán sulfato (Lentza-Rizos \& Avramides, 200 I).

\section{Materiales y métodos}

\section{Ubicación del sitio experimental}

Los ensayos se realizaron en el laboratorio de Entomología del Centro Universitario de Suroccidente, Universidad de San Carlos de Guatemala, que se encuentra ubicado en el municipio de Mazatenango, departamento de Suchitepéquez, Guatemala.

\section{Recolección de hojas y flores de llama del bosque y obtención de extractos}

Los árboles de llama del bosque que se utilizaron para el estudio estaban en una finca cafetalera de Mazatenango y Samayac, Guatemala. Una vez localizado el árbol, se procedió a realizar la recolección de las hojas y flores frescas de apertura reciente, posteriormente se secaron en un horno de convección de aire forzado a $40{ }^{\circ} \mathrm{C}$ (se recolectó I $\mathrm{kg}$ de peso seco) para realizar la extracción en el laboratorio. Las hojas y flores secas se procesaron en el laboratorio de Investigación de Plantas y Productos Naturales (LIPRONAT) de la Facultad de Ciencias Químicas y Farmacia de la Universidad de San Carlos de Guatemala. Se pesó una muestra de $200 \mathrm{~g}$ de hojas secas molidas y $74 \mathrm{~g}$ de flores secas, estas fueron maceradas en etanol al $95 \%$ y se dejaron reposar tres días. Las soluciones etanólicas obtenidas se filtraron y se concentraron a presión reducida en un rotaevaporador marca Buchi, obteniendo un residuo de 17,22 gramos de extracto de hojas y I I,77 g de extracto de flores. 


\section{Tratamientos evaluados $y$ diseño experimental}

Los extractos de hojas y flores se diluyeron para obtener un volumen final de $250 \mathrm{ml}$ de cada concentración $(0,5,1,0$ y I,5 mg/ml de solución). Primero se realizó la disolución del extracto en una solución agua alcohol al 25\%, tomando en cuenta que la relación definida es de I mg de extracto por cada ml de solución. En el testigo relativo se aplicó Endosulfán 35 EC en dosis de 2 l/Ha en 200 I de agua. El testigo absoluto fue la aplicación de etanol-agua destilada al $25 \%$ en volumen mediante un atomizador. El alcohol utilizado fue Etanol al 95\%. Las brocas del café en estado adulto fueron proporcionadas por el laboratorio de la Asociación Nacional de Café, de Guatemala (ANACAFE), donde se realiza la crianza de estos insectos con fines de investigación.

En este ensayo se utilizó un diseño completamente al azar. Se evaluaron cinco tratamientos y tres repeticiones. Debido a las limitaciones de insectos de crianza de laboratorio, para cada tratamiento se utilizaron 30 brocas del café en estado adulto. Los tratamientos $(T)$ se presentan en el Cuadro I.

En el segundo ensayo se aplicaron extractos de flores de llama del bosque en las mismas concentraciones de los primeros tres tratamientos del Cuadro I.

Los tratamientos se aplicaron con un atomizador de I $25 \mathrm{ml}$ de capacidad. Los insectos se mantuvieron alimentados con granos de café en estado pergamino. En el periodo de incubación de los insectos se colocaron en una cámara de aislamiento a temperatura de $27 \pm 1{ }^{\circ} \mathrm{C}$., la humedad relativa fue del $80 \%$. Las lecturas se tomaron a las 24, 48 y 72 horas después de la aplicación de los extractos. Los insectos fueron tocados levemente con un pincel para conocer su condición (muertos o vivos), además se utilizó una lupa para observar si presentaban movimiento.

\section{Variable de respuesta evaluada y análisis de datos}

La variable elegida para los respectivos análisis fue el porcentaje de mortalidad acumulado de broca del café a los tiempos establecidos de evaluación después de la aplicación de los tratamientos. Los datos del porcentaje de mortalidad de insectos fueron transformados mediante la fórmula de arcoseno $\left(V_{x}+0.5\right)$ y posteriormente se realizaron análisis de varianza (ANDEVA) y prueba de medias de Tukey, siempre que las medias presentaran diferencias significativas entre tratamientos al 5\% de significancia, para determinar los tratamientos con los mejores resultados del efecto insecticida de los extractos. El paquete estadístico utilizado fue el "Paquete de diseños experimentales de la Facultad de Agronomía, Universidad Autónoma de Nuevo León (FAUANL), versión 1.4".

\section{Resultados}

Efecto insecticida de extractos de hojas del árbol de llama del bosque sobre la broca del café

En el Cuadro 2 se presentan los porcentajes de mortalidad de brocas del café en sus diferentes lecturas. En la lectura a las 24 horas de la aplicación, había muerto el total de brocas con el insecticida Endosulfán (T4) y solo el 10\% de brocas en el control negativo (T5). En los tratamientos de los extractos, la mortalidad aumentó al utilizarse una mayor concentración de estos, alcanzando el 60\% en el tratamiento T3. A las 48 horas se incrementó la mortalidad de brocas al $50 \%$ en los tratamientos

Cuadro I.Tratamientos utilizados en los ensayos con broca de café.

\begin{tabular}{|c|c|}
\hline Tratamiento & Descripción \\
\hline T1 & Extracto a $0,5 \mathrm{mg} / \mathrm{ml}$ de hojas de llama del bosque \\
\hline T2 & Extracto a $1 \mathrm{mg} / \mathrm{ml}$ de hojas de llama del bosque \\
\hline T3 & Extracto a $1,5 \mathrm{mg} / \mathrm{ml}$ de hojas de llama del bosque \\
\hline T4 & Control positivo (Endosulfán $35 \mathrm{EC}$ ) \\
\hline T5 & Control negativo (etanol + agua al $25 \%)$ \\
\hline
\end{tabular}


Cuadro 2. Mortalidad de brocas del café después de la aplicación de extractos de hojas de llama del bosque.

\begin{tabular}{|c|c|c|c|}
\hline \multirow[b]{2}{*}{ Tratamientos } & Lectura a las $24 \mathrm{~h}$. & Lectura a las 48 h. & Lectura a las $72 \mathrm{~h}$. \\
\hline & Mortalidad \% & Mortalidad \% & $\begin{array}{c}\text { Mortalidad } \\
\%\end{array}$ \\
\hline $\mathrm{TI}$ & 30 & 50 & 60 \\
\hline $\mathrm{T} 2$ & 60 & 60 & 70 \\
\hline T3 & 30 & 50 & 80 \\
\hline $\mathrm{T} 4$ & 100 & 100 & 100 \\
\hline T5 & 10 & 10 & 20 \\
\hline
\end{tabular}

Referencias:Tl: Extracto hojas 0,5 mg/ml,T2: Extracto hojas I mg/ml,T3: Extracto hojas I,5 mg/ml,T4: Endosulfán 35 EC,T5: Etanol +Agua al 25\%.

TI y T3. A las 72 horas la mortalidad acumulada de brocas fue de 60, 70 y $80 \%$ en los tratamientos TI, T2 y T3. La menor mortalidad de brocas se reportó en el control negativo (etanol-agua 25\%), con un $20 \%$ de brocas muertas.

Se realizó un ANDEVA para los resultados del porcentaje de brocas muertas a las 24 horas y se encontraron diferencias estadísticas significativas entre tratamientos, con un 5\% de significancia. Posteriormente, se realizó una prueba de medias al $5 \%$ de significancia y los resultados se presentan en el Cuadro 3.

En los cuadros 2 y 3 se observa que el Endosulfán (T4) fue el más efectivo para provocar la mortalidad de las brocas del café, estadísticamente presenta la media más alta, sin embargo, es igual al T2, T3 y TI. Con la aplicación de Endosulfán se obtuvo el 100\% de mortalidad de broca del café desde las 24 horas después de la aplicación, mientras que con las aplicaciones de extractos la mayor mortalidad fue del $60 \%$ con T2.

Cuadro 3. Prueba de medias de Tukey para el porcentaje de brocas del café muertas a las 24 horas por efecto de los tratamientos.

\begin{tabular}{|c|c|c|}
\hline Tratamiento & \multicolumn{2}{|c|}{ Media } \\
\hline 4 & 3.2400 & $\mathrm{~A}$ \\
\hline 2 & 2.3367 & $\mathrm{AB}$ \\
\hline 3 & 1.7600 & $\mathrm{AB}$ \\
\hline 1 & 1.7267 & $\mathrm{AB}$ \\
\hline 5 & 1.0500 & $\mathrm{~B}$ \\
\hline
\end{tabular}

Transcurridas 48 horas se procedió a realizar otro ANDEVA del porcentaje de brocas muertas y también se encontraron diferencias estadísticas significativas entre los tratamientos evaluados, con un 5\% de nivel de significancia. Se realizó una prueba de medias de Tukey y los resultados se presentan en el Cuadro 4.

En el Cuadro 2 se observa que los resultados del porcentaje de mortalidad de las brocas del café presentan la misma tendencia que la primera lectura. En los tratamientos TI y T3 se incrementó la mortalidad en un $20 \%$, acumulando el $50 \%$ de mortalidad en cada tratamiento de extractos de hojas de llama del bosque. Estadísticamente (Cuadro 4), el Endosulfán (T4) presenta la media más alta, sin embargo, es igual al TI,T2 y T3 pero difiere estadísticamente del control negativo (T5) que presentó el mismo porcentaje de mortalidad de brocas que a las 24 horas (Cuadro 2).

Al término de las 72 horas de aplicación de los tratamientos, se realizó la última lectura y se procedió

Cuadro 4. Prueba de medias de Tukey para el porcentaje de brocas del café muertas a las 48 horas por efecto de los tratamientos.

\begin{tabular}{|c|c|c|}
\hline Tratamiento & \multicolumn{2}{|c|}{ Media } \\
\hline 4 & 3.2400 & $\mathrm{~A}$ \\
\hline 2 & 2.3367 & $\mathrm{AB}$ \\
\hline 1 & 2.2900 & $\mathrm{AB}$ \\
\hline 3 & 2.2233 & $\mathrm{AB}$ \\
\hline 5 & 1.0500 & $\mathrm{~B}$ \\
\hline
\end{tabular}


a hacer otro ANDEVA del porcentaje de brocas muertas. Se encontraron diferencias estadísticas significativas entre los tratamientos evaluados, con un 5\% de nivel de significancia, por lo cual se efectuó una prueba de medias de Tukey y los resultados se presentan en el Cuadro 5.

Los resultados de la prueba de Tukey del Cuadro 5 muestran que el T4,T3,T2 y TI estadísticamente con un nivel de significancia del $5 \%$ son iguales y las medias obtenidas son diferentes. Los tratamientos de extractos de hojas con mayor porcentaje de mortalidad de brocas del café fueron el T3 con el $80 \%$ y el T2 con el 70\% (Cuadro 2). Sin embargo, sigue siendo el Endosulfán (T4) el que presentó el mayor porcentaje de mortalidad, que fue del 100\% desde las primeras 24 horas de la aplicación.

Cuadro 5. Prueba de medias de Tukey para el porcentaje de brocas del café muertas a las 72 horas por efecto de los tratamientos.

\begin{tabular}{|c|c|c|}
\hline Tratamiento & \multicolumn{2}{|c|}{ Media } \\
\hline 4 & 3,2400 & $\mathrm{~A}$ \\
\hline 3 & 2,8900 & $\mathrm{~A}$ \\
\hline 2 & 2,7600 & $\mathrm{AB}$ \\
\hline 1 & 2,5100 & $\mathrm{AB}$ \\
\hline 5 & 1,6767 & $\mathrm{~B}$ \\
\hline
\end{tabular}

En la figura 4 también se presenta el mismo comportamiento de los tratamientos. Se observa que el Endosulfán (T4) provocó la mortalidad del 100\% de las brocas del café desde las 24 horas y este se acumuló hasta las 72 horas de la aplicación. En el caso de los extractos de hojas de llama del bosque, el segundo tratamiento con mayor efecto insecticida fue el extracto de hojas a $1,5 \mathrm{mg} / \mathrm{ml}$ (T3), que provocó la muerte del $80 \%$ de las brocas, y el extracto a $1,0 \mathrm{mg} / \mathrm{ml}$ (T2), que provocó el 70\% de mortalidad en ensayos de laboratorio. En el tratamiento de control negativo (T5), la mortalidad fue de $20 \%$.

\section{Efecto insecticida de los extractos de flores del árbol llama del bosque sobre la broca del café}

La mortalidad de la broca del café en porcentaje por acción del extracto de flores de llama del bosque se observa en el cuadro 6. A las 24 horas de la aplicación, la mortalidad de las brocas del café fue del 100\% con el Endosulfán (T4), también con los tratamientos de extractos T2 y T3, para el caso del TI fue del $70 \%$. Esto indica que los extractos de flores tuvieron mayor efecto insecticida en menos tiempo comparado con los extractos de hojas.

Se realizó un ANDEVA al porcentaje de brocas muertas a las 24 horas y los resultados establecieron diferencias estadísticas significativas entre los tratamientos evaluados, con un 5\% de nivel de

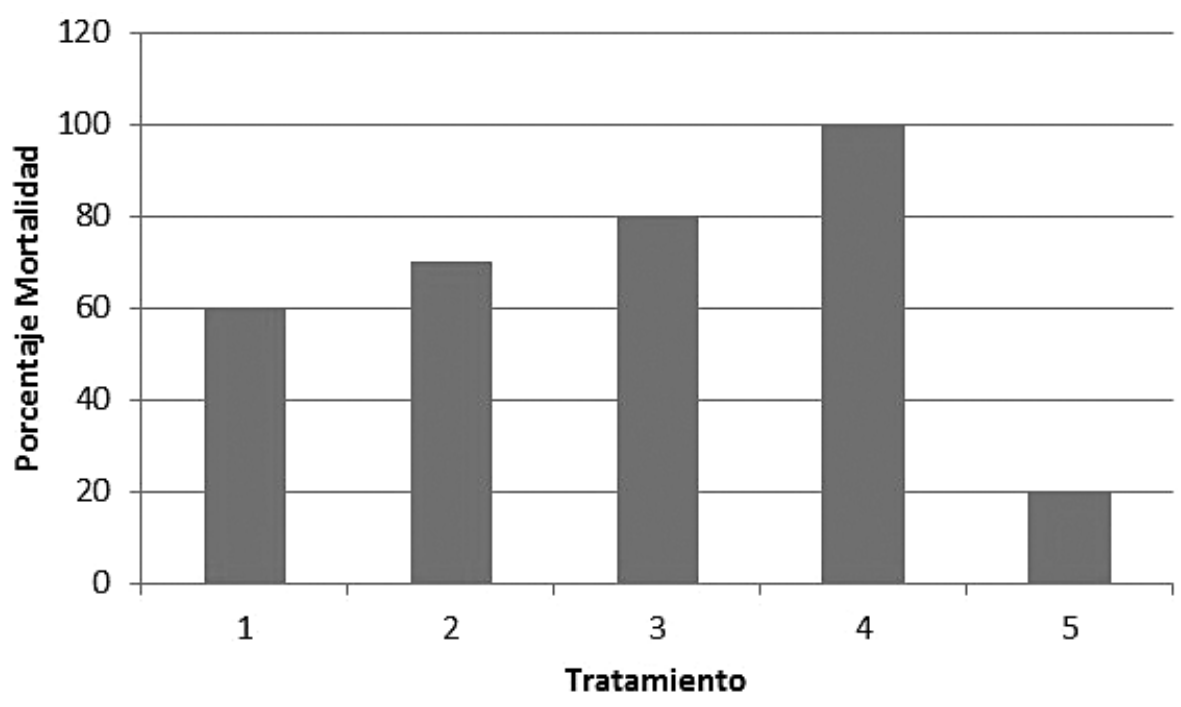

Figura 4. Mortalidad de la broca del café en porcentaje a las 72 horas de aplicación de los extractos de hojas. 
Cuadro 6. Mortalidad de brocas del café después de la aplicación de extractos de flores de llama del bosque.

\begin{tabular}{|c|c|c|c|}
\hline \multirow{2}{*}{ Tratamientos } & Lectura a las $24 \mathrm{h.}$ & Lectura a las $48 \mathrm{~h}$. & Lectura a las $72 \mathrm{h.}$ \\
\hline TI & Mortalidad \% & Mortalidad \% & 70 \\
\hline T2 & 70 & 70 & 100 \\
\hline T3 & 100 & 100 & 100 \\
\hline T4 & 100 & 100 & 100 \\
\hline T5 & 100 & 100 & 40 \\
\hline
\end{tabular}

Referencias:Tl: Extracto flores 0,5 mg/ml,T2: Extracto flores I mg/ml,T3: Extracto flores I,5 mg/ml,T4: Endosulfán 35 EC,T5: Etanol +Agua al 25\%.

significancia. Se realizó una prueba de medias de Tukey y los resultados se presentan en el cuadro 7.

Estadísticamente, el Endosulfán (T4) presenta la media más alta, igual que T2 y T3, en términos estadísticos son iguales a TI. Todos estos tratamientos son diferentes estadísticamente a T5, que forman un segundo grupo. La mortalidad en los tratamientos T4, T2 y T3 fue del 100\%. Esto indica que bajo las condiciones experimentales en las que se realizó el ensayo, los extractos de flores a $\mid$ o $1,5 \mathrm{mg} / \mathrm{ml}$ pueden producir el mismo efecto insecticida que el Endosulfán.

Al transcurrir 48 horas de la aplicación de los tratamientos, se realizó otra lectura y se procedió a realizar un ANDEVA del porcentaje de brocas muertas. Se encontraron diferencias estadísticas significativas entre los tratamientos evaluados, con un $5 \%$ de nivel de significancia, por lo cual se hizo una prueba de medias de Tukey y los resultados se presentan en el Cuadro 8.

Cuadro 7. Prueba de medias de Tukey para el porcentaje de brocas del café muertas a las 24 horas por efecto de los tratamientos.

\begin{tabular}{|c|c|c|}
\hline Tratamiento & Media \\
\hline 2 & 2,3452 & A \\
\hline 3 & 2,3452 & A \\
\hline 4 & 2,3452 & A \\
\hline 1 & 1,8971 & A \\
\hline 5 & 1,1785 & B \\
\hline
\end{tabular}

La prueba de Tukey arrojó casi los mismos resultados estadísticos de los valores de las medias de TI, T2, T3 y T4, solo cambió el valor de T5 porque la mortalidad de las brocas del café aumento del 25 al 40\%. A las 72 horas se presentaron los mismos resultados anteriores y se concluyó el ensayo.

En la Figura 5 se muestra claramente que los extractos de flores de llama del bosque a I y l,5 mg/ml (T2 y T3) fueron tan efectivos como el Endosulfán (T4) y provocaron el 100\% de mortalidad de las brocas del café a las 72 horas de la aplicación bajo condiciones experimentales de laboratorio.

\section{Discusión de resultados}

Los ensayos de laboratorio demostraron que los extractos de hojas del árbol llama del bosque tienen un efecto insecticida. Para el tratamiento TI, el porcentaje de mortalidad de brocas fue de 30,50 y $60 \%$ en las lecturas realizadas a las 24, 48 y 72 horas de aplicación; en elT2 ese porcentaje fue de 60, 60 y $70 \%$ y en el T3 de 30, 50 y $80 \%$, en las tres lecturas de tiempo respectivamente. El mayor porcentaje

Cuadro 8. Prueba de medias de Tukey para el porcentaje de brocas del café muertas a las 48 horas por efecto de los tratamientos.

\begin{tabular}{|c|c|c|}
\hline Tratamiento & \multicolumn{2}{|c|}{ Media } \\
\hline 2 & 2,3452 & $\mathrm{~A}$ \\
\hline 3 & 2,3452 & $\mathrm{~A}$ \\
\hline 4 & 2,3452 & $\mathrm{~A}$ \\
\hline $\mathrm{I}$ & 1,8971 & $\mathrm{~A}$ \\
\hline 5 & 1,5236 & $\mathrm{~B}$ \\
\hline
\end{tabular}




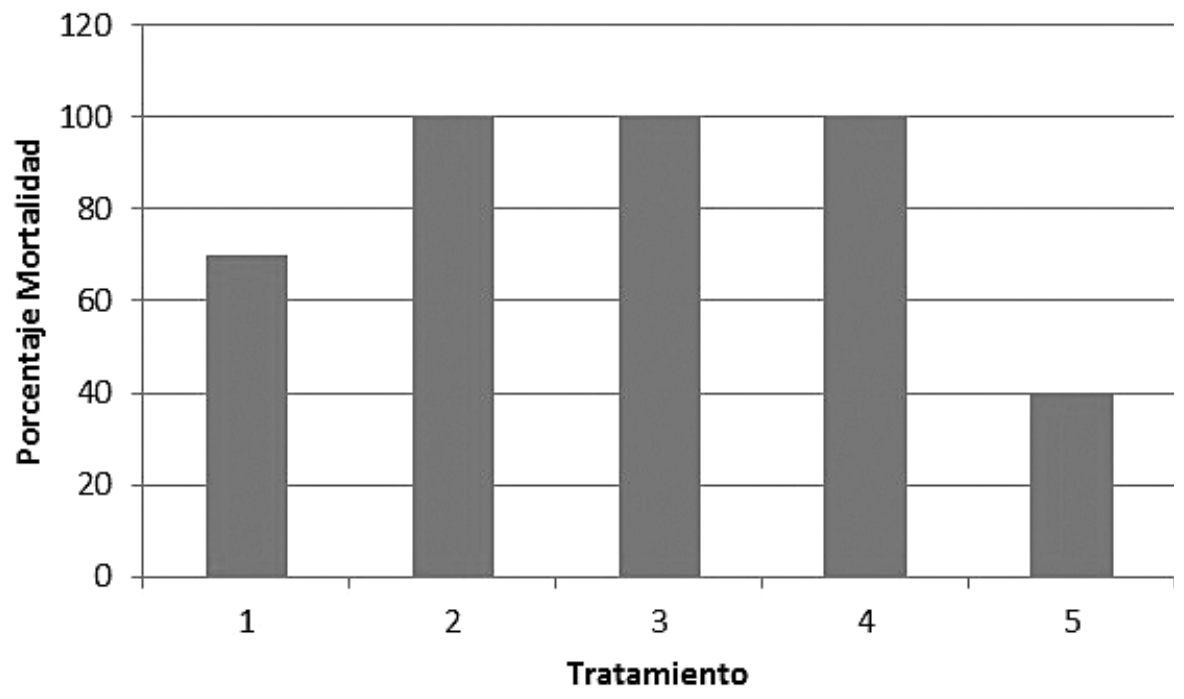

Figura 5. Mortalidad de la broca del café en porcentaje a las 72 horas de aplicación de los extractos de flores.

de mortalidad tras la aplicación de los extractos lo mostró el tratamiento T3, que corresponde a 1,5 $\mathrm{mg} / \mathrm{ml}$. Ninguno de los extractos alcanzó el $100 \%$ de mortalidad producido por el insecticida Endosulfán tras 24 horas de aplicación. Con relación al control negativo (T5), la mortalidad de brocas fue del 10 , 10 y $20 \%$ en las lecturas realizadas respectivamente (cuadro 2).

Con respecto a los extractos de flores, la eficacia de los tratamientos fue la siguiente: T4, T2 y T3 mostraron un 100\% de mortalidad a las 24 horas de la aplicación. Esto indica que bajo las condiciones experimentales en que se realizó el ensayo, los extractos de flores a I ó I,5 mg/ml pueden producir el mismo efecto insecticida que el Endosulfán. Estadísticamente TI, T2 y T3 son iguales pero la mortalidad en el TI solo alcanzó el 70\% para todas las lecturas realizadas. En el control negativo (T5), la mortalidad fue de 25,40 y $40 \%$ en las tres lecturas respectivamente (cuadro 6).

Los resultados del porcentaje de mortalidad de brocas del cafe in vitro muestran la efectividad y el potencial insecticida de los extractos de hojas y flores de llama del bosque y se relacionan con los resultados de actividad insecticida obtenidos por Segantini et al. (2002), quienes evaluaron el efecto de extractos acuosos de las hojas de S. campanulata al I, 2, 3, 4 y 5\% sobre larvas del segundo estado de Spodoptera frugiperda. En este ensayo se realizaron lecturas de mortalidad de larvas a las 24, 36, 48 y 72 horas después del inicio del experimento en laboratorio y demostraron que a las 72 horas las concentraciones de 4 y $5 \%$ causaron una mortalidad del 9 I al $100 \%$. También se relacionan con los ensayos de TorresEstrada et al. (20I0), que evaluaron extractos acuosos al 10 y $5 \%$ de Spathodea campanulata, los cuales provocaron una mortalidad del $89,9 \%$ y $100 \%$ de los estados inmaduros del mosquito Anopheles albimanus en condiciones de laboratorio.

Finalmente, se recomienda evaluar las concentraciones de extractos de I y $\mid, 5 \mathrm{mg} / \mathrm{ml}$ de hojas y flores de llama del bosque en otros ensayos de laboratorio, invernadero o en una plantación comercial de café infestada con broca, con el fin de generar más información sobre la actividad insecticida de los extractos de esta especie de árbol para el control de esta plaga.

\section{Bibliografía}

ANACAFE (1998). Manual de Caficultura. Guatemala:Asociación Nacional del Café, p. I84-187.

Andrews, K. \& Quezada, J. (1989). Manejo Integrado de Plagas Insectiles en Agricultura. Honduras, Escuela Agrícola El Zamorano, p. 3-20.

Davis, A., Pylatuik, J., Poradis, J. \& Low, N.H. ( 1998). Nectar carbohydrate and composition vary in relation to nectar anatomy and location whitin individual flowers of several species of Brassicaceae. Planta, p. 305-318.

Das, A., Dhanobalan, R. \& Doss, A. (2009). In Vitro Antibacterial Activity of Two Medicinal Plants Against Bovine Udder 
Isolated Bacterial Pathogens from Dairy Herds. Ethnobotanical Leaflets 13: 152-158.

Flach, A. (2005). Ecologia química de Maxillariinae, Spathodea campanulata e Meliponninae. Tese Doutorado. Sao Paulo, Universidade Estadual de Campinas, Instituto de Quimica, $202 \mathrm{p}$.

García, J. (1997). Introducción a los plaguicidas. CR: Editorial Universidad Estatal a Distancia, p. I-I3.

Hellpap, C. (2000). El desarrollo de un plaguicida botánico. Pasos necesarios. En: Plantas con potencial biocida. Metodologías y experiencias para su desarrollo. Lima: Editorial Gráfica Sttefany, S.R. Ltda., p. 75-8I.

Hill, D. (2008). Pest of crops in warmer climates and their control. United Kingdom: Springer, p. 232-27l.

Lentza, Ch. \& Avramides, E.J. (200I). Determination of residues of endosulfan and pyrethroid insecticides in virgin olive oil. Journal of Chromatography A, p. 921-927.

Makinde, J., Adesogan, E. \& Amusan, O. (1987). The schizontocidal activity

of Spathodea campanulata leaf extract on Plasmodium berghei berghei in mice. Phytoterapy Research I (2): 65-68.

Makinde, J., Amusan, O. \& Adesogan, E. (1998). Antimamalarial Active of Spathodea campanulata Steam Bark. Planta Medica I0(8): 692-693.

Miller, P.S. (1995). Guía para la identificación de árboles comunes, para técnicos forestales de Guatemala. Manual de USDA Forest Service, p. 74-75.
Ngouela, S., Nyasse, B., Tsamo, E., Sondengam, B. \& Connolly, J. (1990). Spathodic acid: a triterpene acid from the stem bark of Spathodea campanulata. Plant Physiology and Biochemistry 29( I 2): 3959-396 |.

OEA. (2002). Una nueva estrategia de desarrollo para las Américas (desde los derechos humanos y el medio ambiente). OEA/Ser. 6. CP/CAJP-1987/02. Centro de Derechos Humanos y Medio Ambiente, 22 p.

Portugal-Araujo, V. (1963). O perigro de dispersao da Tuliperira do Gabao (Spathodea campanulata Beauv). Chacaras e Quintais 107: 562.

Segantini, B., Romano, F. \& Ribeiro, C. (2002). Efeito insecticida de extracto

aquoso de folhas de Spathodea campanulata (Bignonianceae) sobre Spodoptera frugiperda (J.E. Smith, 1797) Lepidoptera: Noctuidae em Laboratorio. Artigo do Facultade Cantareira, Brasil, s.p.

SEMICOL (2010). Manual Práctico de Manejo de Semillas y Viveros Agroforestales. SEMICOL S.A., 90-92.

Torres-Estrada, J.,Velazquez, J., Delgado, S., Velazquez, M., Navarro, R. \& Rodriguez, A. (2010). Research and Reports in Tropical Medicine 2010: 83-87.

Trigo, J. \& Santos, W. (2000). Insect Mortality in Spathodea campanulata Beauv. (Bignoniaceae) Flowers. Rev. Bras. Biol. 60 (3): 537-538. 